\title{
The behaviour and wellbeing of children and adults with severe intellectual disability and complex needs: The Be-Well checklist for carers and professionals
}

by

Chris Oliver ${ }^{1 *}$, Dawn Adams ${ }^{2}$, Debbie Allen ${ }^{3}$, Hayley Crawford ${ }^{4}$, Mary Heald ${ }^{1}$, Jo Moss ${ }^{5}$, Caroline Richards ${ }^{1}$, Jane Waite ${ }^{6}$, Alice Welham ${ }^{7}$, Lucy Wilde ${ }^{8}$ and Kate Woodcock ${ }^{1}$

${ }^{*}$ Corresponding author. Prof. Chris Oliver, C.Oliver@Bham.ac.uk. School of Psychology, University of Birmingham, Birmingham UK B15 2TT

${ }^{1}$ School of Psychology, University of Birmingham, UK.

${ }^{2}$ Autism Centre of Excellence, Griffith University, Australia.

${ }^{3}$ Faculty of Education Health \& Wellbeing, University of Wolverhampton, UK.

${ }^{4}$ Warwick Medical School, University of Warwick, UK

${ }^{5}$ School of Psychology, University of Surrey, UK

${ }^{6}$ School of Life \& Health Sciences, Aston University, UK

${ }^{7}$ Department of Neuroscience, Psychology and Behaviour, University of Leicester, UK.

${ }^{8}$ Faculty of Arts \& Social Sciences, The Open University, UK

Please use this citation:

Oliver, C., Adams, D., Allen, D., Crawford, C., Heald, M., Moss, J., Richards, C., Waite, J., Welham, J., Wilde, L., and Woodcock, K. (In press). The behaviour and wellbeing of children and adults with severe intellectual disability and complex needs: the Be-Well checklist for carers and professionals, Paediatrics and Child Health, https://doi.org/10.1016/j.paed.2020.09.003 


\begin{abstract}
Children and adults with severe intellectual disability and complex needs often show behaviours and distress that carers and professionals find difficult to identify causes for, manage and decrease. The prevailing view is that these behaviours and distress are learned and consequently interventions focus on behavioural techniques. In this article we summarise the findings of research that indicate that behaviour and distress in this population are influenced by transient and stable characteristics or conditions that can either interact with aspects of learning, be independent of learning, and interact with each other. These transient and stable characteristics or conditions are: pain and discomfort, sensory sensitivity, anxiety and low mood, sleep problems, atypical emotional regulation, specific cognitive difference, and differences in social behaviour. To aid carers and professionals, we present a checklist of the elements of an assessment process that covers these transient and stable characteristics or conditions and other relevant influences on behaviour and distress such as seizures, medication, learning and communication. We also draw attention to the benefit of identifying the cause of intellectual disability to inform the assessment process.
\end{abstract}




\section{Introduction}

In this article we describe the transient and stable characteristics or conditions that influence the behaviour and wellbeing of children and adults with severe intellectual disability and complex needs. We present these influences in a checklist format, with homage to Atul Gawande, for use across settings, by professionals and carers ${ }^{1}$. The purpose of this article is to draw attention to areas that should be considered during assessment, formulation and intervention, because the evidence base and clinical experience indicate that these areas: 1 ) exert direct and indirect influences on behaviour and wellbeing, 2) can usually be modified via existing interventions and 3) are typically neglected in this population.

The population of interest is defined by profound or severe intellectual disability, minimal or no verbal communication, and often additional physical, sensory, cognitive and social impairment or atypicality. The cause of intellectual disability within this population is notably heterogeneous and includes genetic syndromes for a large proportion of individuals, alongside other pre-, peri- and post-natal factors. The clinical presentation is extremely diverse, as is any behavioural presentation that may prompt referral. Importantly, the same class of behavioural presentation (for example, selfinjury, distress or aggression) can have different causes within and between children, hence the need for a checklist. Additionally, we believe that use of a checklist reduces the likelihood of diagnostic overshadowing, whereby compromised wellbeing or behaviours are erroneously considered to be the inevitable results of (or even to be definitive of) severe intellectual disability and complex needs.

\section{A checklist of influences on behaviour and distress.}

There are seven person characteristics or conditions that we propose are influential. These are: 1) pain and discomfort, 2) sensory impairment or sensitivity, 3) anxiety, 4) sleep problems, 5) atypical emotion regulation, 6) specific cognitive difference (over and above intellectual disability) and 7) atypical social behaviour. Two further influences are driven more by the environment and, whilst they interact with these seven characteristics or conditions, they are also fundamentally important in themselves. These are: 1) the learned function of behaviours and 2) expressive communication skills. Typically, more than one of the listed characteristics or conditions will be experienced by any child with severe intellectual disability and complex needs, and each can also interact with other areas, either in a causal chain or by enhancing other influences. It is the accumulation of the characteristics and conditions, and their additive or interactive influences, which accentuate the clinical complexity and challenge. In the description of each influence below, we describe causal chains and interactions.

By proposing the checklist, we do not argue for or against the use of psychiatric diagnoses such as autism, and attention deficit hyperactivity disorder. This is because the characteristics and conditions that we propose as influential cut across diagnoses and are more precise for the purpose of intervention. For example, although inattention, impulsivity and hyperactivity comprise diagnostic features of ADHD, only impulsivity appears in our checklist and hence might warrant intervention in this context. It is possible that a psychiatric diagnosis can help access to evidence based interventions in appropriate services delivered by experienced practitioners, although children with severe intellectual disability and complex needs are often excluded from these services.

The use of the checklist

\footnotetext{
${ }^{1}$ The checklist is available in the appendices.
} 
The checklist is intended for use to address both behaviour and distress specifically, and the individual's and family wellbeing more generally. It has value: 1 ) when there is a specific problem identified by carers, 2) for identifying risk for future behaviour and distress and 3) during periodic reviews of people with severe intellectual disability and complex needs. It is important to note that the focus of this article is on person characteristics and conditions that influence behaviour and wellbeing. The focus is not on broad environmental influences such as family relationships, resilience, carer availability, caregiver familiarity with behavioural interventions or service availability, each of which is nevertheless important. These are covered and emphasised in the majority of overviews of interventions. The article and checklist are intended to redress an imbalance by drawing attention to conditions and characteristics that are often overlooked in assessment and formulation and which interact with environmental influences. They are additional, not alternative, targets for intervention.

\section{Developing the checklist}

The checklist has been constructed for use by anyone involved in the support of children and adults with severe intellectual disabilities and complex needs. We propose its use by professionals across educational, social and clinical services, and by parents and carers to ensure that the areas are kept under review throughout development, as different areas become more or less prominent at different times and because more effective interventions may become available with time.

To construct the checklist, we identified characteristics and conditions that have been demonstrated to influence behaviour, distress and wellbeing in published literature. Often this literature is at an early stage with large scale studies across people with different causes of intellectual disability yet to be conducted. Whilst the strength of evidence is therefore variable, we would argue that proof of principle is sufficient to warrant assessment and that the ethical imperative of identifying possible causes of the most harmful behaviours should take precedence at this point. A good example is headbanging to the point of open wounds and/or bruising that is caused by pain from an unidentified but treatable health condition such as gastro-oesophageal reflux. There are no definitive large scale studies of this association but tangential evidence and clinical experience is compelling. In each section of the checklist we have not necessarily included detailed information on the specific causes of a condition (for example sleep problems) or the range and sequence of assessments. We believe decisions at this level are best made on a case by case basis. The purpose of the checklist is to guide attention toward each area, rather than prescribe how assessment, formulation and interventions are conducted.

The majority of the evidence that we draw upon when identifying a characteristic or condition as influential is derived from our own research. Much of this evidence comes from the study of the relationship between specific genetic syndromes and behavioural, emotional and cognitive outcomes. This research strategy for understanding individual influences on behaviour, distress and wellbeing enabled us to identify groups for which a condition or characteristic was relatively stable and common and compare these groups with other groups for which this was not the case. This also enabled examination of within group variability to identify influences on outcome variables. The purported influence identified with this strategy could then be tested in another group, and then in the wider population of individuals with intellectual disabilities. For each influence, this process of identifying commonalties that cut across causes of intellectual disability is at a different stage. At this point, we believe that for each of the listed influences, there is sufficient proof of principle within at least one syndrome group to justify identifying the influence as warranting attention and assessment. 


\section{Assumptions underpinning the checklist}

As noted above, the checklist is neutral with regard to the value of psychiatric diagnosis. Equally, for checklist items, the cause of intellectual disability per se does not explain why behaviours or a state of wellbeing are evident, even though they might be more common in one syndrome than another. This reasoning would be diagnostic overshadowing writ large. The merit of the identification of a specific genetic cause of intellectual disability is that it will alert practitioners and carers to the likelihood, nature and atypical presentation of some specific checklist items that influence behaviour and wellbeing. Hence, assessment priorities can be identified. Importantly, for each checklist item the degree of intellectual disability should not be invoked to explain the presence of a characteristic or condition. Intellectual disability should be (is) defined by the discrepancy between both adaptive behaviour and intellectual performance, and chronological age. It follows then that any other characteristics or conditions have a different explanation, just as they would for a child who does not have an intellectual disability. This premise will help avoid diagnostic overshadowing and interpreting the association between a greater degree of intellectual disability and the presence characteristics and conditions as directly causal rather than correlational. In other words, for children with severe intellectual disability and complex needs, the characteristics and conditions should be assessed and treated just as they would be for typically developing children. To do otherwise is, arguably, discriminatory.

\section{Enhancing the value of the checklist}

We propose four things that will enhance the use of the checklist. The first is the identification of the cause of intellectual disability. This does not serve the purpose of explaining behaviour and wellbeing but will increase awareness of likely influences on behaviour and wellbeing and help prioritise assessments. As noted above, the majority of known genetic neurodevelopmental syndromes are associated with a number of the characteristics and conditions identified here. Organisations from which good quality and immediately relevant information on specific syndromes is available include Contact A Family, individual syndrome support group websites, Society for the Study of Behavioural Phenotypes, GeneReviews and the Further Inform Neurogenetic Disorders (FIND) and UNIQUE websites. The second is the regular review of possible seizures and medication use. The third is the process of clearly identifying and defining the behaviours and $\backslash$ or distress that might be differentiated from and influenced by the characteristics and conditions. The emphasis should be on precise but workable terms that enable shared identification of the agreed therapeutic goals for identified behaviour and \or distress. Terms such as "challenging behaviour" are too imprecise to be useful and often conflate the characteristics and conditions that influence behaviour and the behaviour itself. The $\mathrm{CBI}$ and $\mathrm{CBQ}$ can be used to precisely identify behaviours and their severity, while the MIPQ can assess distress ${ }^{2}$. The fourth is the use of simple outcome measures. Each defined and identified behaviour and $\backslash$ or distress, and the relevant characteristics or conditions, should be assessed at first presentation. This might be via a five point scale (or similar) or a more established measure (CBI, CBQ or MIPQ repeated), usually comprising informant completed rating scales. Assuming that these measures are adequately sensitive to change (a factor which must be considered from the outset), their use may allow a relatively objective assessment of progress over time from a baseline.

The checklist: Person characteristics and conditions

\footnotetext{
${ }^{2}$ Assessments mentioned in the article are listed in the appendices.
} 
1. Pain and discomfort. There is growing evidence that pain and discomfort relate directly to distress and pervasive low mood, and also to behaviours such as self-injury and poor sleep, while having an indirect effect on other behaviours such as aggression. Additionally, health conditions, such as middle ear infections, skin conditions and gastro-intestinal disorders, are associated with self-injury in autistic children, with pain and discomfort the likely direct cause. Gastro-intestinal disorders such as reflux and constipation are common causes of distress and self-injury, and reflux can cause middle ear infections and tooth decay. Some potentially painful health conditions are associated with specific syndromes, such as hip dislocation and blepharitis in Cornelia de Lange syndrome, reflux associated with scoliosis in Angelman syndrome, tumours in Tuberous Sclerosis Complex and peripheral sensory neuropathy and middle ear infections in Smith-Magenis syndrome.

There are four mechanisms by which pain and discomfort might influence behaviour: 1) the behaviour serves the purpose of attempting to remove the painful stimulus, such as when an individual inserts their fingers into their throat as a response to reflux, 2) pain-gating, by which a behaviour, such as hair pulling, 'gates' or blocks transmission of other painful stimuli, 3) pain, via routes 1 or 2, can cause the first instances of behaviour that can then be subjected to social reward (a checklist item) and 4) an interaction with a learned behaviour (a checklist item). For example, demands in a classroom are experienced as more unpleasant when pain is experienced and so a learned escape behaviour is more likely and more rewarding. Pain can also disrupt sleep onset and sleep maintenance (a checklist item) and aspects of disorders such as reflux can be exacerbated in the presence of anxiety (a checklist item) and vice versa.

Assessment of pain and discomfort is difficult in nonverbal or minimally verbal children with complex needs. Typically, to convey that they are in pain, a child of typical development might identify the perception of pain, label the pain, understand that communicating about pain is important and then accurately impart a description of the sensation; all processes which may be compromised for non/minimally-verbal children. The most likely indicators in this population may be changes from a stable baseline in a cluster of behaviours (mood and self-injury for example) and changes in sleep. Additionally, it is important to note that the usual indicators of a painful condition seen in typically developing children might not be displayed. Parents and carers are often very reliable at identifying pain as they are aware of the change from baseline in their child's behaviour and sensitive to alternative explanations of behaviour. Pain related behaviour is often described as 'out of the blue' and unrelated to the environment, associated with distress evidenced by vocalisation or facial expression, and episodes of behaviour are unlikely to be stopped by carer intervention. As noted previously, diagnostic overshadowing is a concern, with pain-related behaviour automatically attributed instead to a syndrome or autism, for example. Two useful assessments of pain are the FLACC and the Non-Communicating Child Pain Checklist. Interventions are unlikely to be different from those for typically developing children. The most common problems reported by parents are lack of acknowledgement that a child is in pain and reluctance to refer to specialists. Clearly, regular physical health and dental checks will prove beneficial.

\section{Sensory impairment or sensitivity. Impaired vision and compromised peripheral nerve} transmission can each be directly related to behaviour. For example, if the optic tract and occipital lobe are unaffected but there is no response to light, then eye pressing and rubbing can occur due to the rewarding effect of perceived light. The sensation and perception of pain are poorly understood in children with severe intellectual disability and complex needs, but there is evidence of some possible atypicalities. It is suggested, for example, that individuals with syndromes such as SmithMagenis and Prader-Willi may have a higher pain threshold. Consequently, self-injury in individuals with these syndromes may not have immediately negative consequences, and might therefore be 
more severe than would otherwise be the case. It is unclear whether unusual pain perception is equally evident for both acute and chronic pain.

Under-and over-response to sensory stimuli have attracted attention recently, with increased emphasis in criteria for autism diagnosis, and reports of atypicalities in a number of syndrome groups. A higher pain threshold has been included as "under-responsiveness", but is perhaps a special case, with clear implications for behaviour that exposes children to dangers such as scalding water. Over-responsiveness is, arguably, more problematic given its propensity for precipitating behaviour and distress. There is evidence of hyperacusis in autism, as well as in a number of syndromes (Smith-Magenis, fragile $X$ and Williams, amongst others). Hyper-responsiveness to light and texture are also reported in autism. Hyper-responsiveness can develop later in some syndromes. In Smith-Magenis and Cornelia de Lange syndromes, early hearing impairments are common such that the development of hyperacusis is delayed. Consequently, sensory sensitivity should be evaluated across the lifespan, with attention to hyper- and hyposensitivity across and within modalities.

Hyper-responsiveness to stimuli is, almost by definition, directly associated with distress as evidenced by emotional reaction such as covering ears, removing clothing with labels, and refusing to eat foods with specific textures. Stimuli can also evoke episodes of behaviour that can then be rewarded by removal of the stimuli, or of the person from the stimuli. In this way behavioural responses, such as self-injury or disrupting the environment, can become learned through operant conditioning (a checklist item). Additionally, if environments or events are associated with aversive sensory stimuli, then anticipation of exposure to the environment or event can lead to the developmental of anxiety and/or a phobia of environments/events (a checklist item). Consequently, early awareness of hyper-responsiveness can help prevent the development of later problems. Responsiveness to sensory stimuli can be assessed using the Sensory Profile, and Sensory Processing Measure. Interventions are in their infancy but include modulating sensory input, for example using headphones and ear defenders for hyperacusis. Other psychological interventions, such as systematic desensitisation, are in development.

3. Anxiety and low mood. There is growing evidence that anxiety is common in children with severe intellectual disability and complex needs. Across the lifespan, the prevalence of anxiety disorders is between 11 and $84 \%$ in autism, and ranges from $40 \%$ to $80 \%$ in genetic syndromes. Importantly, anxiety in people of typical development might differ from anxiety in autism and the type of anxiety disorder experienced differs across neurodevelopmental disorders (including genetic syndromes). In autism, the most common forms are specific phobias, in fragile $X$ syndrome social anxiety and specific phobias are prominent, in Williams syndrome phobias and generalised anxiety disorders are common whilst in Cornelia de Lange syndrome specific phobias and separation anxiety prevail. Social anxiety is perhaps a special case and is considered below (Social behaviour). It should be noted that the typical forms and manifestation of anxiety disorders may differ in children with severe intellectual disability and complex needs, and autism specifically.

Whilst there may be underlying central nervous system structural and functional atypicalities driving anxiety, these have yet to be identified in children with severe intellectual disability and complex needs. There is preliminary evidence for psychological mechanisms that might account for anxiety, with implications for prevention and intervention. The first is the classical and operant conditioning (two factor) model of phobias (described above in relation specifically to sensory hypersensitivity). The second is intolerance of uncertainty, which is found to be heightened in people with anxiety disorders in the context of typical development. Intolerance of uncertainty refers to a fundamental propensity to find unpredictability anxiety provoking. In autism, it is argued that many repetitive 
behaviours and restricted interests occur as adaptive responses to unpredictable situations, in order to lower aversive levels of arousal and provide some predictability or routine. Other psychological mechanisms that might account for anxiety include compromised executive function (see below, specific cognitive difference) and hypervigilance for anxiety provoking stimuli. There are a number of possible consequences for behaviour and distress that arise from these mechanisms. First, if environments and events are experienced as anxiety provoking, then behaviours might emerge that lead to an environment or event being avoided (for example, displaying aggression prior to going to school may lead to a delay in arriving at school). Second, if there is an interruption to repetitive behaviours and restricted interests employed as mechanisms of anxiety reduction, then anxiety will increase, and this might evoke a behavioural response. Third, repeated exposure to high levels of (i.e. frequent or intense) uncertainty can lead to low mood and decreased interest and pleasure. Arguably, the negative effects of exposure need to be balanced by the benefits of building tolerance to uncertainty by gradually increasing the amount of exposure to uncertainty. As for pain and discomfort, anxiety is difficult to assess and specific behaviours and reported indicators of increased psychophysiological arousal, or freezing and withdrawal, in specific situations may be the best indicator. Avoidance behaviours and hypervigilance may both be prominent for some stimuli, and behaviour may change as events become closer. Typical signs of anxiety such as sweating, tense stance and pale appearance might be evident, but there are autonomic system atypicalities in some syndromes that might mean some signs are not evident. It is also important to note that some behavioural signs of distress might overlap with those that indicate pain, and also that anxiety can interact with reflux and hence increase pain (a checklist item).

Some behavioural interventions with established efficacy for some types of anxiety (e.g., exposurebased interventions for specific phobias) may sometimes be considered in some cases, and adaptations used for people with moderate intellectual disability and autism might be beneficially applied.

4. Sleep problems. Poor sleep is common in children with intellectual disability. The prevalence of sleep problems is higher in those with genetic syndromes, and autistic children, and hence issues with sleep are more likely in children with severe intellectual disability and complex needs. The causes of sleep problems are varied and a comprehensive review is beyond the scope of this article. Particular sleep problems are related to different neurodevelopmental disorders and genetic syndromes. For instance, short sleeping and high variability in night-to-night sleep is prominent in Angelman syndrome, settling problems are common in autism, obstructive and central sleep apnoea are evident in Down and Prader-Willi syndromes and an inverted melatonin release cycle causes night-time waking and daytime sleepiness in Smith-Magenis syndrome. There is also evidence that pain (a checklist item) can disrupt sleep, as can anxiety (a checklist item), and that some sleep problems can be learned behaviours (a checklist item) and can be related to sensory sensitivity (a checklist item).

Disrupted sleep is related to daytime behaviour, with the relationship likely mediated by atypical emotion regulation or irritability (a checklist item). Additionally, sleep might be related to behaviour via compromised behavioural inhibition (a checklist item), i.e., the capacity of a child to regulate their own behaviour. For example, sleep apnoea in Down syndrome is related to poor inhibition, with treatment for apnoea now advised. There is good reason to think this relationship also exists in other children. Sleep disorders in children with intellectual disability are related to parental stress and hence capacity to deal with distress and behaviour on a day to day basis. Interestingly, it is not necessarily just the amount of sleep loss that is important, with unpredictability and fragmentation of sleep also influential. 
Assessing sleep can be problematic, as parent reports might only indicate when parents (as opposed to children) are awake. Some standardised assessments such as the BISQ, MSPSQ and the CSHQ can indicate broad areas that warrant further assessment. Gold standard assessments such as polysomnography are very difficult for most children with severe intellectual disability and complex needs. However, actigraphy is now often possible, readily available and valid, with modified scoring protocols and commercially available sleep trackers worn on wrists or under bedsheets. The evidence for therapeutic use of melatonin in this population is very limited and focussed on autism, and a trial of weighted blankets for autistic children did not show a positive outcome. Interventions for sleep disorders are unlikely to differ substantively from those for typical children, with surgical correction of obstructive sleep apnoea and behavioural intervention well documented with evidence of effectiveness.

5. Emotional dysregulation. Behaviours such as 'temper' or emotional outbursts have typically been conceptualised as "learned" in children with intellectual disability, and assessments and interventions are hence often similar to those for behaviours such as disrupting the environment, aggression and screaming. However, there is evidence that emotional outbursts might be better conceptualised as indicative of emotional dysregulation in this population. The prevalence of emotional outbursts varies across genetic syndromes; for instance, they are particularly prominent in Prader-Willi, Smith-Magenis and Lowe syndromes and persist well beyond early childhood. Such variability across syndromes is not consistent with a conceptualisation of the outbursts as purely learned behaviours. Additionally, after emotional outbursts people with Prader-Willi syndrome exhibit extreme remorse, perhaps indicative of compromised control, and during the outbursts there is evidence of autonomic hyperarousal with facial flushing and increased salivation. Early trials of vagal nerve stimulation in Prader-Willi syndrome are encouraging.

Emotional outbursts are typically evoked by environmental triggers, such as disruption to routine (related to a checklist item; specific cognitive difference) and denial of food in Prader-Willi syndrome, with some different triggers more influential in Smith-Magenis and Lowe syndromes and others highly influential across syndromes. Interestingly, disruption to routine commonly evokes a different emotional response of anxiety (a checklist item) in fragile $X$ syndrome. This suggests that whilst the threshold for emotional response to the same environmental trigger is lowered in both syndromes, the form of the emotional response can differ. Assessment of emotional regulation in children with severe intellectual disability and complex needs is in its infancy and there is a clinical need to differentiate episodes of emotional dysregulation from learned behaviours (a checklist item), with implications for management and intervention. Parents often report that the best strategy in response to an emotional outburst is to ensure the child is safe and wait for the episode to abate, although co-regulation strategies can sometimes help. Identifying triggers for outbursts is important and implementing strategies to limit the effect of triggers can be beneficial.

6. Cognitive difference. Specific cognitive difference in children with severe intellectual disability is rarely considered important. However, there is growing evidence that specific elements of cognitive difference in people with intellectual disability, over and above general intellectual disability, might underpin behaviours that pervade everyday life and can predispose to more difficult behaviours, distress, anxiety (a checklist item) and emotional states (a checklist item). For example, across neurodevelopmental disorders, problems with inhibition (the ability to supress a behaviour), set shifting (the ability to switch the focus of attention that manifests as impaired cognitive flexibility or an inability to respond differently to the same situation) and working memory have been associated with stronger adherence to routine, more repetitive questioning and greater social anxiety. 
Impulsivity is, in part, a behavioural manifestation of compromised inhibition and a risk marker for aggression in children with intellectual disability, whist repetitive behaviour is, arguably, a manifestation of cognitive difference across a number of areas and a risk marker for self-injury. Impulsivity is also a risk marker for the severity and persistence of self-injury and/or aggression in autism and syndromes such as Tuberous Sclerosis Complex. Compromised set shifting underpins adherence to routine in Prader-Willi syndrome and perhaps other neurodevelopmental disorders. The clinical importance of each of these observations is that specific and combined cognitive differences over and above generalised intellectual disability can: 1) make unavoidable everyday demands more aversive (for example, a trip to the supermarket) 2) interact with other characteristics and conditions to increase the severity of a behavioural outcome (for example a higher pain threshold, poor expressive communication), 3) give insight into the lived experience of children with severe intellectual disability (for example, experiencing an exciting event, such as a party, as anxiety provoking) and 4) change the attributions of others with regard to the cause of behaviours (for example, children who are strongly adherent to routine are unable, rather than stubbornly unwilling, to accept change).

Assessment of these cognitive differences is complex and at a clinical level it is more efficient to identify the presence of the indicative behaviours. The TAQ and RBQ have been designed for use with this population. Adherence to routine, insistence on sameness, and poor impulsivity (for example, evidenced by children who self-injure and who actively restrict their own movements) are good examples of behaviours that are likely related to cognitive difference.

For intervention there is emerging evidence that implementing 'fuzzy' routines, particularly early in life, might be beneficial to prevent routines becoming too established. If routines are unavoidably interrupted, then a unique cue or sign signalling change to routine prior to the change can help children prepare for disruption. Impulsivity can be ameliorated using behavioural techniques that increase tolerance for delay. For children who self-injure and restrict their own movements (using 'self-restraint' behaviours that are associated with severity of self-injury, such as winding the hands into clothing), the method of restriction can be modified gradually to retain the control but not be otherwise restrictive.

7. Social behaviour. There are two areas of social difference that are important for behaviour and distress. The first is the impact of social anxiety on social behaviour. This is evident in a number of neurodevelopmental disorders, such as Cornelia de Lange and fragile $\mathrm{X}$ syndromes and autism. It typically emerges in early adolescence, but can do so much earlier in fragile $X$ syndrome. As for pain and anxiety disorders generally, behaviour is often the only indicator of this area of difficulty in children who are nonverbal or minimally verbal. Avoidance of social situations can manifest as escape behaviours, and repeated exposure can result in distress. Interventions include anxiety management strategies such as systematic desensitisation. The second crucial area of social difference is atypically strong motivation for social contact, evident in some genetic syndromes such as Williams, Angelman and Smith-Magenis. In Angelman syndrome the strong drive for social contact appears to be generalised and may partially depend on the precise genetic cause, whilst in Smith-Magenis syndrome the drive appears to be more specific to a primary caregiver in any given environment.

Importantly, for Angelman and Smith-Magenis syndromes there is evidence that aggression and selfinjury can occur because of the attention that inevitably follows these behaviours, with the strong drive for social contact in these syndromes enhancing the potency of this social reward. For Angelman syndrome, there is evidence that aggression that leads to attention can be decreased using behavioural intervention, and that children can learn to discriminate between times when 
adult attention is available and when it is not. There is clearly a role for advising carers about the strong social reward for both these syndromes at an early stage, to mitigate longer terms problems. This is particularly important given the propensity for sleep problems (a checklist item) in these syndromes, and the possibility of behavioural factors becoming additional causes. Assessment of the social drive can be conducted using informant based measures, such as the SQID.

\section{The checklist: Learned behaviour and communication}

There are two checklist items that straddle person characteristics and the environment and should always feature in assessment.

1. The learned function of behaviours. This checklist item differs from others because it interacts with every other checklist item. For any behaviour, it is important to establish if the behaviour is learned, i.e., occurs because it is triggered by an environmental event or a motivational state and is rewarded, usually, but not always, by a response from others. Such behaviours are called functional and there are different functions to behaviours. The most common functions are reward by attention or more tangible responses (such as the presentation of activities or food) and escape from unwanted or aversive situations, such as demands to engage in a task or go somewhere. A different type of function is sensory reward, such as in the example of eye pressing rewarded by perceived light given above.

There is a wealth of evidence that behaviours such as self-injury, aggression, disruption of the environment and screaming can be functional. It is also possible in some cases that this is the only reason for a behaviour, and that none of the other checklist items is relevant. However, in children with severe intellectual disability and complex needs, given the propensity to experience one or more of the person characteristics or conditions in the checklist, it is likely that any functional behaviour is also influenced by those characteristics or conditions. Examples of this are given for each checklist item above. The characteristics and conditions interact with functional behaviours by: 1) causing the behaviour to start initially, before it then becomes functional (e.g. pain or a behaviour that occurs during a temper outburst such as self-injury), 2) making a motivational state that triggers behaviours more likely (e.g. anxiety, low levels of social contact in some syndromes, disruption to routine being experienced as aversive, experiencing sensory stimuli as aversive), 3) increasing how aversive a social trigger is (for example, demands to do a task may be more aversive for a child who is in pain or sleep deprived) and 4) increasing the likelihood of a behaviour occurring in the presence of a trigger (e.g. in a child with poor behavioural inhibition, the child cannot suppress the behaviour when a trigger such as a motivational state occurs).

A functional assessment of behaviour conducted by a behaviour specialist or clinical psychologist includes informant based measures, such as the QABF, and \or observation of normally occurring events or events occurring during structured situations (experimental functional analysis). Interventions include behaviour management strategies, which typically aim to increase adaptive behaviours (particularly communication, a checklist item), and decrease less adaptive behaviours, using the principles of operant conditioning. It is important to note that these strategies are likely to be significantly more effective if other relevant person characteristics and conditions are addressed at the same time.

It has become widespread clinical practice to assess the functions of behaviour first in any clinical setting, or to assume a behaviour is functional and thus implement interventions or give behaviour management advice based on that assumption. We propose that assessing the functions of behaviour should take place after or alongside the assessment and appraisal of the role of other 
checklist areas. This is because the functional assessment might not be necessary if the cause of behaviour is identified as pain, for example, and also because assessment of areas such as anxiety, sensory hypersensitivity, and cognitive and emotional difference can indicate atypical functions for behaviours.

2. Communication. The last checklist item differs from others as it is probably protective with regard to functional behaviours and distress. There is substantial evidence that functional behaviours are analogous to expressive communication, in that they can influence the behaviour of others to satisfy a motivational state. Notably, children with severe intellectual disability and complex needs will have, almost by definition, compromised expressive communication, and this might increase the likelihood that a behaviour will attain function.

For the purpose of decreasing behaviour and distress, the development of a comprehensive or sophisticated expressive communication system is not the primary aim. For children with severe intellectual disability and complex needs, there should be an absolute minimum communicative repertoire that includes the ability to request: that an activity is started, stopped or paused, removal from a situation, and that social contact, food and drink, and help are provided.

In proposing that these areas of basic communication of needs are assessed, we are not suggesting that other areas of communication are unimportant or do not warrant intervention. Rather, effective communication in these areas is more likely than others to have a pivotal impact on behaviour and distress. Assessment of these areas can be done formally using standardised social situations; however, for clinical purposes, informally identifying a child's ability to communicate these basic needs across different environments or with different communication partners is critical.

Should basic communication skills need to be taught or made more effective, then the form of communication is not necessarily important and can be objects, signs, cards, simple electronic devices or vocalisations. The most important thing is that communication can be understood by all the people the individual needs or wants to interact with, that they are always with the person and they are responded to quickly and reliably.

Conclusions: "... grant me the serenity to accept the things I cannot change, the courage to change the things I can, and the wisdom to know the difference." Reinhold Niehbuhr.

The checklist we have proposed covers the areas in which we believe intervention can yield the greatest benefit, most efficiently, for children with severe intellectual disability and complex needs, with regard to behaviour and distress. As noted in the introduction, we have not covered areas and interventions that are exclusively environmental, as we wanted to ensure emphasis on child characteristics and conditions, since these are so often neglected. We are sure that the checklist will be modified in the future, as areas prove more or less influential and evidence changes. However, it is intended to provide a useful starting point in its explicit documentation of our current understanding of the most prominent influences on behaviour and distress. As noted in the introduction, the checklist can be used to guide clinical evaluation in response to referral, in order to focus on areas that might prevent later problems and to ensure relevant factors are reviewed periodically. It is also applicable to adults with severe intellectual disability and complex needs. Our hope is that the checklist enables practitioners and empowers carers to focus systematically and collaboratively on active assessment, formulation and intervention that best serves wellbeing in the prevailing service context. 


\section{Bibliography}

Cooper V, Emerson E, Glover G, et al. Early intervention for children with learning disabilities whose behaviour challenges. Briefing Paper. Challenging Behaviour Foundation (2014).

Gawande, A. (2010). The Checklist Manifesto: How to Get Things Right. New York, Metropolitan Books.

Waite, J., Heald, M., Wilde, L., Woodcock, K., Welham, A., Adams, D. and Oliver, C. (2014). The importance of understanding the behavioural phenotypes of genetic syndromes associated with intellectual disability. Paediatrics and Child Health. 10, 468-472.

Woodcock, K. A. and Blackwell, S. (2020). Psychological treatment strategies for challenging behaviours in neurodevelopmental disorders: what lies beyond a purely behavioural approach? Current Opinion in Psychiatry, 33, 92-109. 


\section{Assessments}

\section{Defining and assessing behaviour and wellbeing (CBI, CBQ, MIPQ)}

Oliver, C., McClintock, K., Hall, S., Smith, M., Dagnan, D. \& Stenfert-Kroese, B. (2003). Assessing the severity of challenging behaviour: Psychometric properties of the Challenging Behaviour Interview. Journal of Applied Research in Intellectual Disabilities, 16, 53-61.

https://www.findresources.co.uk/professionals/login

Hyman, P., Oliver, C. \& Hall, S. (2002). Self-injurious behavior, self-restraint and compulsive behaviors in Cornelia de Lange syndrome. American Journal on Mental Retardation, 107, 146-154.

https://www.findresources.co.uk/professionals/login

Ross, E. \& Oliver, C. (2003). Preliminary analysis of the psychometric properties of the Mood, Interest and Pleasure Questionnaire (MIPQ) for adults with severe and profound learning disabilities. British Journal of Clinical Psychology, 42, 81-93.

https://www.findresources.co.uk/professionals/login

\section{Pain and discomfort. (FLACC and NCCPC)}

Merkel, S.I., Voepel-Lewis, T., Shayevitz, J.R. and Malviya, S. (1997). The FLACC: a behavioral scale for scoring postoperative pain in young children. Pediatr Nurs. 23, 293-297.

(https://cerebra.org.uk/download/flacc-pain-scale-infographic/)

Breau, L.M., Finley, G.A., McGrath, P.J. and Camfield, C.S. (2002). Validation of the Noncommunicating Children's Pain Checklist-Postoperative Version. Anesthesiology, 96, 528-535.

http://www.community-networks.ca/wp-content/uploads/2015/07/PainChklst_BreauNCCPCR2004.pdf

\section{Sensory difference (Sensory Profile)}

Dunn W. (2014). Sensory Profile 2: User's Manual. USA: Pearson, Inc..

\section{Anxiety}

Esbensen, A.J., Rojahn, J., Aman, M.G. et al. (2003). Reliability and Validity of an Assessment Instrument for Anxiety, Depression, and Mood Among Individuals with Mental Retardation. J Autism Dev Disord 33, 617-629.

\section{Sleep problems (BISQ, MSPSQ, CSHQ)}

Sadeh, A. (2004).A brief screening questionnaire for infant sleep problems: validation and findings for an Internet sample. Pediatrics. 113:e570-e577. doi:10.1542/peds.113.6.e570

https://pediatrics.aappublications.org/content/pediatrics/113/6/e570.full.pdf

Simonds JF, Parraga H. (1982). Prevalence of sleep disorders and sleep behaviors in children and adolescents. J Am Acad Child Adolesc Psychiatry. 21,383-8.

Owens, J.A., Spirito, A., McGuinn, M., (2000). The Children's Sleep Habits Questionnaire (CSHQ): Psychometric Properties of A Survey Instrument for School-Aged Children. Sleep, 23, 1-9. https://doi.org/10.1093/sleep/23.8.1d 


\section{Emotion dysregulation}

Shields, A., \& Cicchetti, D. (1997). Emotion regulation among school-age children: The development and validation of a new criterion Q-sort scale. Developmental Psychology, 33, 906-916.

https://depts.washington.edu/dbpeds/Screening\%20Tools/CHSQ\%20article.pdf

\section{Cognitive difference (TAQ, RBQ)}

https://depts.washington.edu/dbpeds/Screening\%20Tools/CHSQ\%20article.pdf

The Activity Questionnaire (TAQ).

Burbidge, C., Oliver, C., Moss, J., Arron, K., Berg, K., Hill, L., Trusler, K., Furniss, F. and Woodcock, K. A. (2010). The association between repetitive behaviours, impulsivity and hyperactivity in people with intellectual disability. Journal of Intellectual Disability Research, 54, 1078-1092. DOI: 10.1111/j.13652788.2010.01338.x

https://www.findresources.co.uk/professionals/login

The Repetitive Behaviour Questionnaire (RBQ).

Moss, J., Oliver, C., Arron, K., Burbidge, C. and Berg, K. (2009). The prevalence and phenomenology of repetitive behavior in genetic syndromes. Journal of Autism and Developmental Disorders.39, 572588. (DOI: 10.1007/s10803-008-0655-6)

https://www.findresources.co.uk/professionals/login

\section{Social Behaviour (SQID)}

Sociability Questionnaire for Intellectual Disability (SQID).

Moss, J., Nelson, L., Powis, L., Waite, J. and Oliver, C. (2016). A comparative study of sociability and selective mutism in autism spectrum disorder, Angelman, Cri du Chat, Cornelia de Lange, fragile $X$ and Rubinstein Taybi syndromes. American Journal on Intellectual and Developmental Disabilities, 121, 465-486.

https://www.findresources.co.uk/professionals/login

\section{Learned function of behaviours (QABF).}

Paclawskyj, T.R., Matson, J.L., Rush, K.S., Smalls, Y. and Vollmer, T.R. (2000). Questions about behavioral function (QABF): a behavioral checklist for functional assessment of aberrant behavior. Res Dev Disabil. 21, 223-229. doi:10.1016/s0891-4222(00)00036-6

\section{Communication}

Kiernan, C. and Reid, B. (1987). Pre-Verbal Communication Schedule https://www.mosaiccommunication.com.au/pvcs 


\title{
The Be-Well. The Behaviour and Wellbeing checklist for children and adults with severe intellectual disability and complex needs
}

\section{The Be-WeLL}

\author{
The cause of intellectual disability
}

(note associated characteristics)

\begin{tabular}{|l|l|}
\hline $\begin{array}{l}\text { Behaviours of concern or indicators of distress (specify, } \\
\text { e.g. headbanging, self-biting, scratching, screaming, } \\
\text { dropping to floor, crying, unable to be still, shouting, } \\
\text { groaning). Indicate frequencylseverity. }\end{array}$ & \\
\hline Medication review & $\begin{array}{l}\text { Specific purpose of medication. Last review date. } \\
\text { Monitoring of therapeutic and side effects. }\end{array}$ \\
\hline Seizure investigation & Screening for seizures. \\
\hline
\end{tabular}

\begin{tabular}{|c|c|}
\hline $\begin{array}{l}\text { Area to } \\
\text { consider/review? }\end{array}$ & Consider presence. Seek formal assessment if in evidence \\
\hline $\begin{array}{l}\text { Pain and } \\
\text { discomfort }\end{array}$ & $\begin{array}{l}\text { Facial expression (two lines in forehead), crying \groaning\screaming, inconsolable, } \\
\text { frequent leg movements, unable to be still, defends a body area. Behaviour or negative } \\
\text { emotion occurs out of the blue. Parent reports change in these behaviours or is } \\
\text { concerned about possible pain. } \\
\text { Review/note: Dates of last dental, gastro-intestinal (especially reflux and constipation), } \\
\text { middle ear investigations. Health conditions related to cause of intellectual disability. }\end{array}$ \\
\hline $\begin{array}{l}\text { Sensory } \\
\text { impairment and } \\
\text { sensitivity }\end{array}$ & $\begin{array}{l}\text { Moves away from, resists, refuses to be in the presence of, or avoids noises, textures, } \\
\text { lights, temperatures. } \\
\text { Review/note: Dates of last vision and hearing investigations. }\end{array}$ \\
\hline Anxiety & $\begin{array}{l}\text { Moves away from, resists, refuses to be in or avoids situations or events, seeking } \\
\text { reassurance, clinging. Appears fearful or tense (muscle tension, pallor). Endures } \\
\text { situation/event with fear, tension and/or distress. }\end{array}$ \\
\hline Sleep & $\begin{array}{l}\text { (As appropriate for age). Night waking, early waking, settling difficulties, co-sleeping } \\
\text { with parent, daytime sleepiness, apnoea, snoring, parasomnias. }\end{array}$ \\
\hline $\begin{array}{l}\text { Emotion } \\
\text { regulation }\end{array}$ & $\begin{array}{l}\text { (As appropriate for age). Emotional outbursts disproportionate to cause. Crying, } \\
\text { screaming, facial flushing, excessive salivation, distress, unable to calm self or be } \\
\text { calmed. }\end{array}$ \\
\hline $\begin{array}{l}\text { Cognitive } \\
\text { difference }\end{array}$ & $\begin{array}{l}\text { 1. Compromised cognitive flexibility as evidenced by: strong adherence to routine, } \\
\text { resistant to change, adverse response to change, checking, tidying, arranging items. } \\
\text { 2. Compromised inhibition as evidenced by: unable to control own behaviour, strong } \\
\text { negative reaction to being thwarted, cannot easily wait, needs an instant response. }\end{array}$ \\
\hline Social behaviour & $\begin{array}{l}\text { 1. Strong preference for adult presence (caregiver or other). Reacts negatively to } \\
\text { absence of social contact. } 2 \text {. Moves away from, refuses to be with, or avoids social } \\
\text { contact with others (especially unfamiliar others) or social events. }\end{array}$ \\
\hline \multirow[t]{2}{*}{$\begin{array}{l}\text { Learned function } \\
\text { of behaviour }\end{array}$} & $\begin{array}{l}\text { Behaviour is triggered by specific events such as: being asked to do something, go } \\
\text { somewhere, stop an activity, someone moving away from the person or not attending } \\
\text { to them, being refused something, approaching or entering a specific environment. } \\
\text { Behaviour typically stops when event that triggered the behaviour stops or is changed. }\end{array}$ \\
\hline & Minimal communication skills. Seek intervention if not demonstrated. \\
\hline Communication & $\begin{array}{l}\text { Across people and places, is able to reliably and effectively request: } 1 \text { ) that an activity is } \\
\text { started, stopped or paused, and 2) change to or removal from a situation (event and 3) } \\
\text { that social contact, food and drink, activities, and help are provided. }\end{array}$ \\
\hline
\end{tabular}

